02,05

\title{
Механизмы, определяющие гистерезис магнитосопротивления гранулярного ВТСП в присутствии парамагнитного вклада, на примере $\mathrm{HoBa}_{2} \mathrm{Cu}_{3} \mathrm{O}_{7-\delta}$
}

\author{
(C) С.В. Семенов ${ }^{1,2}$, Д.М. Гохфельд ${ }^{1,2}$, К.Ю. Терентьев ${ }^{1}$, Д.А. Балаев ${ }^{1,2, \text { ฯ }}$ \\ ${ }^{1}$ Институт фризики им. Л.В. Киренского ФИЦ КНЦ СО РАН, \\ Красноярск, Россия \\ ${ }^{2}$ Сибирский фредеральный университет, \\ Красноярск, Россия \\ ๑ E-mail: dabalaev@iph.krasn.ru
}

Поступила в Редакцию 14 мая 2021 г.

В окончательной редакции 14 мая 2021 г.

Принята к публикации 25 мая 2021 г.

\begin{abstract}
Исследовано гистерезисное поведение магнитосопротивления $R(H)$ гранулярного высокотемпературного сверхпроводника (ВТСП) $\mathrm{HoBa}_{2} \mathrm{Cu}_{3} \mathrm{O}_{7-\delta}$. Сверхпроводники семейства ҮВСО с редкоземельным элементом, имеющим магнитный момент ( $\mathrm{Nd}, \mathrm{Ho}, \mathrm{Er}, \mathrm{Sm}, \mathrm{Yb}, \mathrm{Dy})$, на месте иттрия, характеризуются значительным парамагнитным вкладом в общую намагниченность. Основной целью данной работы было установление возможного влияния этого парамагнитного вклада на магнитотранспортные свойства, которые определяются туннелированием носителей сверхпроводящего тока через межгранульные границы. Анализ полученных результатов в рамках концепции эффективного поля в межгранульной среде показал, что распределение линий магнитной индукции от парамагнитных моментов имеет принципиально иной характер, чем для мейсснеровских токов и абрикосовских вихрей. Линии магнитной индукции от парамагнитных моментов не концентрируются в области межгранульных границ, и, тем самым, оказывают незначительное влияние на магнитотранспортные свойства гранулярного ВТСП. В то же время, в межгранульных границах происходит сильная концентрация линий магнитной индукции, вызванных мейсснеровскими токами и вихрями Абрикосова, в силу специфики их свойств. Именно сжатие магнитного потока, определяет магнитотранспортные (в том числе, гистерезис $R(H)$ ) свойства гранулярных ВТСП, в том числе для структуры 1-2-3 с редкоземельными ионом, обладающим магнитным моментом.
\end{abstract}

Ключевые слова: гранулярный ВТСП $\mathrm{HoBa}_{2} \mathrm{Cu}_{3} \mathrm{O}_{7-\delta}$, парамагнитный вклад, гистерезис магнитосопротивления, эффективное поле, межгранульные границы.

DOI: 10.21883/FTT.2021.10.51392.114

\section{1. Введение}

Объяснение магнитотранспортных свойств гранулярных высокотемпературных сверхпроводников (ВТСП) основывается на известном факте реализации в этих объектах двухуровневой сверхпроводящей системы [1-3]. Рассматриваемые „уровни“ - это ВТСП-гранулы, являющиеся „сильной“ сверхпроводящей подсистемой, и межгранульные границы, сверхпроводящие свойства в которых значительно подавлены, и перенос сверхпроводящего тока через них осуществляется благодаря эффекту Джозефсона. Достаточно большое количество работ по гранулярным ВТСП было посвящено определению механизмов диссипации в подсистеме межгранульных границ во внешнем магнитном поле [4-15]. Однако, зачастую в подобных исследованиях подсистема межгранульных границ, где происходит диссипация, считалась независимой, и при анализе не принимался во внимание необратимый характер зависимостей магнитосопротивления $R(H)$ [16-25], критического тока $I_{\mathrm{C}}(H)$ [26-29], a также температурных зависимостей $R(T)$ [30-35], измеренных во внешнем магнитном поле. Объяснение такого нетривиального поведения магнитотранспортных характеристик уже потребовало принятие во внимание взаимодействия подсистем „гранул““ и „межгранульных границ“.

В разное время были предложены различные модели проникновения магнитного поля в межгранульную среду [36], в ВТСП-гранулы [37-40,18-21], и также рассматривалось перераспределение линий магнитной индукции в межгранульной среде [41]. Логично, что внешнее поле проникает сначала в подсистему межгранульных границ. Величина такого поля проникновения достаточно мала, например, для иттриевой системы это доли Эрстед при азотной температуре и единицы Эрстед при более низких температурах. Проникновение магнитного потока в гранулы происходит уже при бо́льших величинах внешнего поля: порядка десятков Эрстед при азотной температуре и сотен Эрстед при более низких температурах. Однако основной интересной особенностью оказалось влияние магнитных моментов ВТСП гранул на результирующее поле в межгранульных границах.

Рассмотрим указанное влияние подробнее. Намагниченность сверхпроводника определяется мейсснеров- 


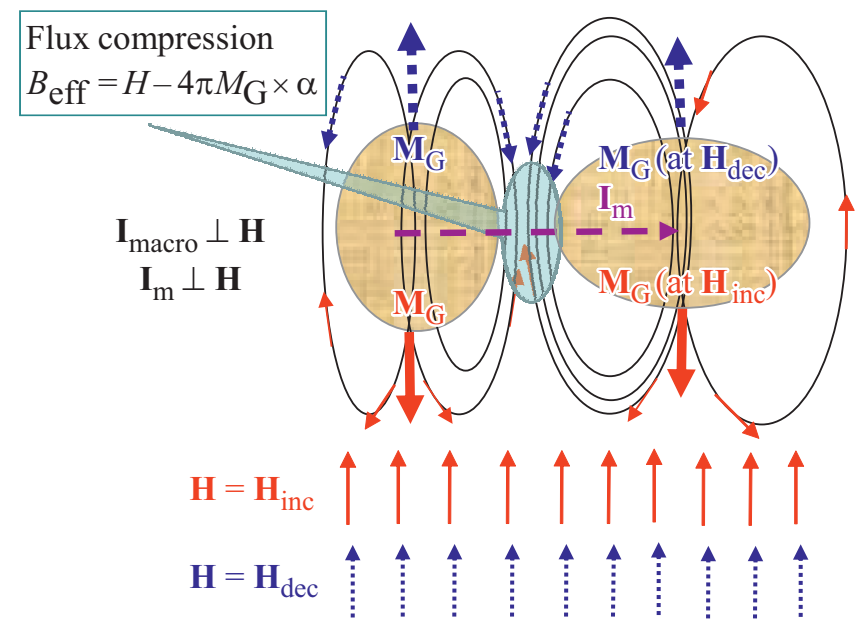

Рис. 1. Схематическое представление распределения линий магнитной индукции от магнитных моментов $\mathbf{M}_{\mathrm{G}}$ ВТСП гранул. Гранулы - овалы, пространство между ними - межгранульная граница, $\mathbf{I}_{\mathrm{m}}-$ микроскопический ток (туннелирование из левой гранулы в правую), $\mathbf{I}_{\text {macro }}-$ макроскопический ток через образец $\left.\mathbf{I}_{\text {macro }} \perp \mathbf{H}\right)$. Направления $\mathbf{M}_{\mathrm{G}}$ и линий магнитной индукции относительно внешнего поля показаны сплошными $\left(\mathbf{H}=\mathbf{H}_{\text {inc }}\right.$, поле возрастает $)$ и пунктирными $\left(\mathbf{H}=\mathbf{H}_{\text {dec }}\right.$, поле убывает) стрелками.

скими токами (они обеспечивают диамагнитное поведение) и захваченным потоком (абрикосовскими вихрями). Суперпозиция указанных вкладов приводит к типичной петле магнитного гистерезиса, для которой намагниченность $M$ принимает отрицательные значения при возрастающем внешнем поле $\left(H=H_{\text {inc }}, H_{\text {inc }}>0\right)$ и положительные значения при убывающем внешнем поле $\left(H=H_{\mathrm{dec}}, H_{\mathrm{dec}}>0\right)$. Соответственно, магнитные моменты гранул $\mathbf{M}_{\mathrm{G}}$ направлены против внешнего поля при $H=H_{\text {inc }}$ и вдоль $\mathbf{H}$ при $H=H_{\text {dec. }}$ Применительно к гранулярному ВТСП можно прийти к картине перераспределения линий магнитной индукции в межгранульных границах, качественно показанной на рис. 1 для двух соседствующих ВТСП гранул. Носители сверхпроводящего тока (на рис. 1 это микроскопический ток $\left.\mathbf{I}_{\mathrm{m}}\right)$ туннелируют через границу между гранулами, и при этом туннелирование происходит в суммарном поле, являющемся суперпозицией внешнего поля Н и поля, индуцированного магнитными моментами $\mathbf{M}_{\mathrm{G}}$. Рассмотрение взаимного направления Н и линий магнитной индукции в межгранульной границе (рис. 1) приводит к тому, что при $\mathbf{I}_{\mathrm{m}} \perp \mathbf{H}$ для суммарного, или эффективного поля $B_{\text {eff }}\left(\mathbf{I}_{\mathrm{m}} \perp B_{\text {eff }}\right)$ будет справедливо выражение [29,42]:

$$
B_{\text {eff }}=H-4 \pi M_{\mathrm{G}} \times \alpha .
$$

Коэффициент $\alpha$ включает в себя средний размагничивающий фактор этих гранул и отражает сгущение силовых линий в межгранульном промежутке из-за сжатия потока. На возможность реализации сжатия магнитного по- тока было впервые указано в работе [41]. Если усреднить коэффициент $\alpha$, уже по всем межгранульным границам (где $\left.\mathbf{I}_{\mathrm{m}} \perp \mathbf{H}\right)$, а также оперировать с намагниченностью материала $\left(M(H)=\Sigma M_{\mathrm{G}}(H)\right)$, то для эффективного поля $B_{\text {eff }}$ в межсгранульной среде получим выражение, подобное (1). Для процессов диссипации знак $B_{\text {eff }}$ (или направление $\mathbf{B}_{\text {eff }}$ относительно $\left.\mathbf{H}\right)$ уже не важен, и в результате выражение (1) перепишется в виде

$$
B_{\text {eff }}(H)=|H-4 \pi M(H) \times \alpha| .
$$

C помощью выражения (2) можно воспроизвести форму гистерезиса магнитосопротивления $R(H)$, используя экспериментальные данные по намагниченности и принимая $R(H) \sim B_{\text {eff }}(H)$ [42-44]. Выражение (2) также описывает форму гистерезиса $I_{\mathrm{C}}(H)$, если считать, что $I_{\mathrm{C}} \sim 1 / R$. Детальное сопоставление экспериментальных зависимостей $R(H)$ с зависимостями $B_{\text {eff }}(H)$ позволило определить, что значение параметра $\alpha$ (выражение $(2))$ достаточно большое: $\alpha \approx 20-25$ для ориентации $\mathbf{H} \perp \mathbf{I}_{\text {macro }}\left(\mathbf{I}_{\text {macro }}-\right.$ направление макроскопического тока), и $\alpha \approx 12-14$ для ориентации $\mathbf{H} \| \mathbf{I}_{\text {macro }}$ [42-45,35]. Это позволило говорить о сильном влиянии сжатия магнитного потока в межгранульной среде (см. рис. 1). Оказалось, что для ВТСП иттриевой системы величина параметра $\alpha$ практически не зависит от температуры (от температур, близких к критической до 4.2 K) [46-49] и не сильно варьируется для образцов, обладающих различной токонесущей способностью (плотностью критического тока) [50]. Использование эффективного по-

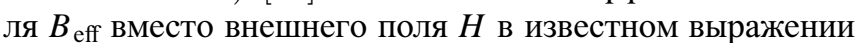
$R=f(H)$ (где $f-$ функция аррениусовского типа) позволило количественно описать экспериментальные гистерезисные зависимости $R(H)$, а также зависимости $R(T)$ (при $H=\mathrm{const}$ ) [51]. Таким образом, вышеописанная концепция эффективного поля в межгранульной среде с учетом сжатия потока хорошо объясняет наблюдаемое поведение диссипации во внешних полях.

Выражение (2) оперирует с макроскопической характеристикой - удельной намагниченностью $M(H)$ гранулярного ВТСП материала. И здесь встает интересный вопрос о том, как будет влиять дополнительная магнитная подсистема в ВТСП гранулах на транспорт носителей сверхпроводящего тока через межгранульные границы. Объектом для таких исследований могут служить гранулярные ВТСП со структурой 1-2-3 с полным или частичным замещением редкоземельного элемента („классического“ иттрия) на магнитный ион. В ВТСП-системах $R e_{1} \mathrm{Ba}_{2} \mathrm{Cu}_{3} \mathrm{O}_{7-\delta}(R e-$ редкоземельный элемент) наличие магнитного момента у $R e(\mathrm{Nd}$, $\mathrm{Ho}, \mathrm{Er}, \mathrm{Sm}, \mathrm{Yb}, \mathrm{Dy})$ практически не влияет на температуру сверхпроводящего перехода $T_{\mathrm{C}}$. Однако наблюдается значительное изменение вида петель магнитного гистерезиса $M(H)$, если $R e$ обладает магнитным моментом [52-56]. Хотя магнитное упорядочение $R e$ подсистемы происходит при температурах не выше $3 \mathrm{~K}$ [57], выше этой температуры $R e$ подсистема 
демонстрирует значительный парамагнитный вклад в общую намагниченность ВТСП образца. При этом, как отмечалось $[52,56]$, вклады от сверхпроводящей и $R e$ подсистем можно считать, практически, независимыми. В данной работе мы исследовали магнитосопротивление гранулярного ВТСП $\mathrm{HoBa}_{2} \mathrm{Cu}_{3} \mathrm{O}_{7-\delta}$ с целью установления того как влияет парамагнитная подсистема на эффективное поле в межгранульной среде.

\section{2. Эксперимент}

Исследованный образец ВТСП $\mathrm{HoBa}_{2} \mathrm{Cu}_{3} \mathrm{O}_{7-\delta}$ был приготовлен методом твердофазного синтеза из соответствующих оксидов с тремя промежуточными помолами. Согласно результатам сканирующей электронной микроскопии (данные были получены на электронном микроскопе Hitachi-TM 4000), средний размер гранул составил 3-4 $\mu \mathrm{m}$.

Для магнитотранспортных измерений использовался четырехзондовый метод. Из полученной таблетки приготавливался образец размерами $0.14 \times 0.2 \times 7 \mathrm{~mm}^{3}$; транспортный ток I был направлен вдоль длинной оси образца. Внешнее поле задавалось электромагнитом при измерениях $R(T)$ выше $77 \mathrm{~K}$ и сверхпроводящим соленоидом при измерениях $R(H)$ при $T=4.2 \mathrm{~K}$. В обоих случаях внешнее поле было перпендикулярно макроскопическому транспортному току $\left(\mathbf{H} \perp \mathbf{I}_{\text {macro }}\right)$. Зависимости $R(H)$ при температуре $4.2 \mathrm{~K}$ были измерены при непосредственном помещении образца в жидкий гелий. Это позволило избежать разогрева образца из-за тепла, выделяющегося на контактах при протекании тока величиной 100-200 mA.

Магнитные свойства (зависимости $M(H)$ и $M(T)$ ) были измерены на вибрационном магнетометре [58] при внешних условиях (включая скорость изменения внешнего поля), соответствующих измерениям $R(H)$. Часть магнитных измерений проведена на установке LakeShore VSM 8604. Перед измерениями образец охлаждался в нулевом внешнем поле.

\section{3. Результаты и обсуждение}

\section{1. Сверхпроводящие и магнитные свойства $\mathrm{HoBa}_{2} \mathrm{Cu}_{3} \mathrm{O}_{7-\delta}$}

На рис. 2 приведены зависимости $R(T)$ исследованного образца, полученные в полях 10,100 и 1000 Ое. Начало сверхпроводящего перехода (резкое уменьшение сопротивления) происходит при температуре $\approx 92.5 \mathrm{~K}$, что совпадает с данными магнитных измерений (при $H=10 \mathrm{Oe}$ ), приведенными на вставке рис. 2. Видимый на рис. 2 двухступенчатый характер зависимостей $R(T)$ и сильное влияние относительно небольших полей на уширение „плавной“ части резистивного перехода типичны для гранулярных ВТСП [1,5,6,12,29,30,35,47-51,59-63]. Такое поведение

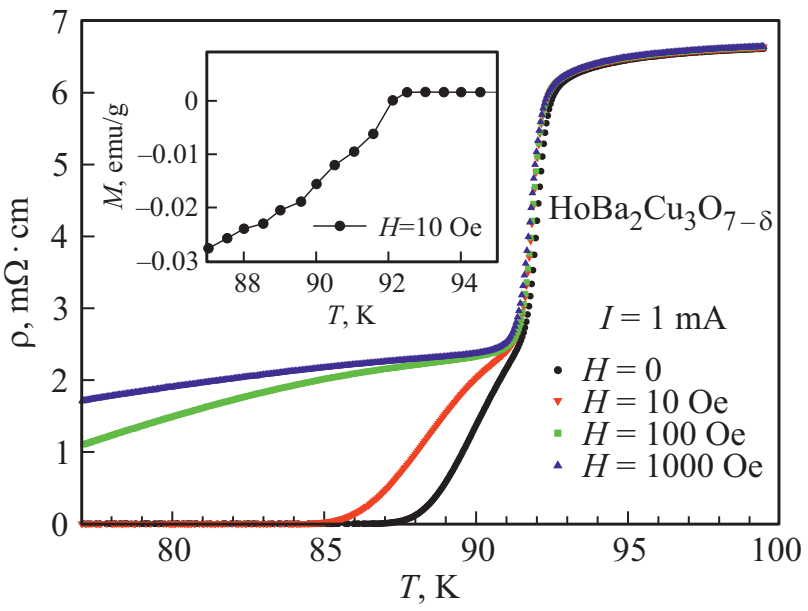

Рис. 2. Зависимости $R(T) \mathrm{HoBa}_{2} \mathrm{Cu}_{3} \mathrm{O}_{7-\delta}$ в различных внешних полях. На вставке: зависимость намагниченности от температуры в поле $H=10$ Ое в окрестности $T_{\mathrm{C}}$.

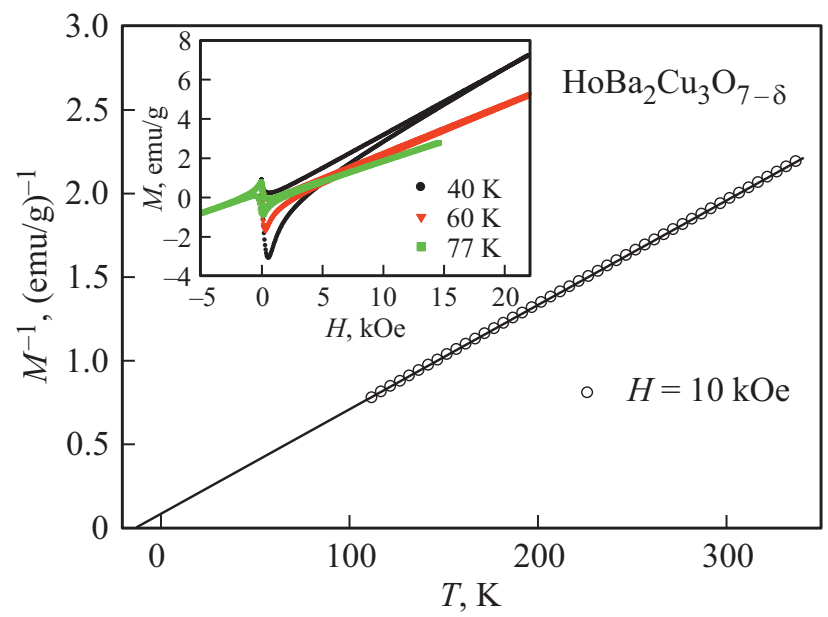

Рис. 3. Зависимость обратной намагниченности от температуры $M^{-1}(\mathrm{~T}) \mathrm{HoBa}_{2} \mathrm{Cu}_{3} \mathrm{O}_{7-\delta}$ в поле $H=10 \mathrm{kOe}$ в области температур выше $T_{\mathrm{C}}$ (символы). Прямая сплошная линия указывает на выполнение закона Кюри-Вейсса, см. п. 3.1. На вставке: участки петель магнитного гистерезиса при указанных температурах $\left(T<T_{\mathrm{C}}\right)$.

зависимостей $R(T)$ отражает наличие двух сверхпроводящих подсистем, как было указано во Введении. Очевидно, что плавная часть резистивного перехода соответствует диссипации в подсистеме межгранульных границ.

На рис. 3 приведена температурная зависимость намагниченности (в координатах $M^{-1}$ от $T$ ), измеренная в поле $H=10 \mathrm{kOe}$ в области температур выше $100 \mathrm{~K}$. Как видно из рисунка, зависимость $M(T)$ исследованного $\mathrm{HoBa}_{2} \mathrm{Cu}_{3} \mathrm{O}_{7-\delta}$ демонстрирует парамагнитное поведение: экспериментальные данные укладываются на линейную зависимость $M^{-1} \sim T$, показанную прямой линией. Видно, что данная линейная зависимость пересекает ось $T$ в отрицательной области температур, 


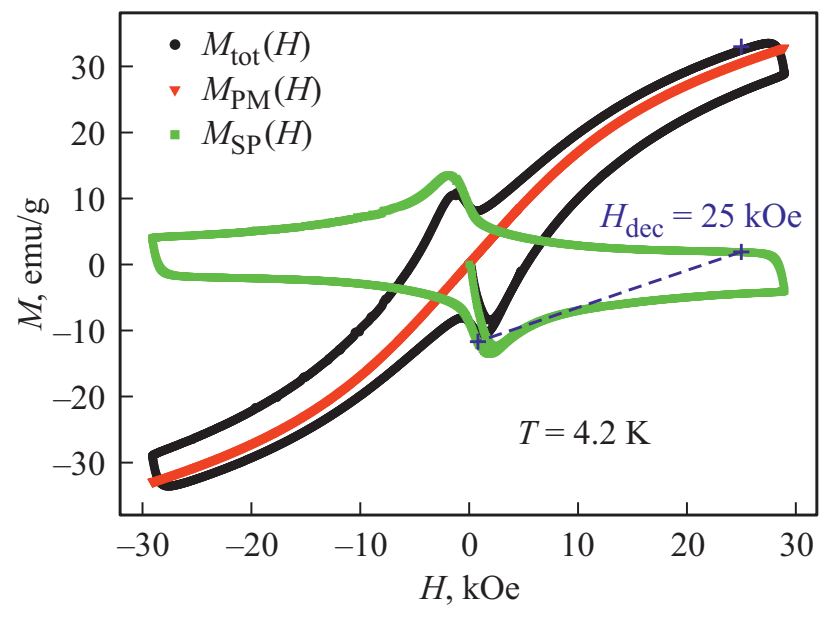

Рис. 4. Петля магнитного гистерезиса $M_{\text {tot }}(H)$ образца $\mathrm{HoBa}_{2} \mathrm{Cu}_{3} \mathrm{O}_{7-\delta}$ при $T=4.2 \mathrm{~K}$ и разделенные парамагнитный $M_{\mathrm{PM}}(H)$ (см. п. 3.1) и сверхпроводящий $M_{\mathrm{SP}}(H)$ вклады, см. п. 3.1. Обозначенные „плюсами“ и соединенные пунктирной прямой точки на зависимости $M_{\mathrm{SP}}(H)$ - значения намагниченности (при $H_{\mathrm{dec}}=25 \mathrm{kOe}$ и $H_{\mathrm{inc}}=1.1 \mathrm{kOe}$ ), соответствующие условию $R\left(H_{\mathrm{dec}}=25 \mathrm{kOe}\right)=\mathrm{const}$ для зависимости $R(H)$ на рис. $5, a$, см. п. 3.2 .

что свидетельствует об антиферромагнитом характере упорядочения магнитных моментов гольмия. Соответствующая температура Кюри-Вейсса составляет $-13 \mathrm{~K}$. Вставка рис. 3 иллюстрирует участки петель магнитного гистерезиса при температурах, соответствующих области существования сверхпроводимости. Помимо сверхпроводящего отклика отчетливо проявляется парамагнитный вклад (видимый наклон зависимостей $M(H)$ в достаточно больших полях), который уменьшается с температурой. Наибольший вклад от подсистемы магнитных моментов гольмия наблюдается при $T=4.2 \mathrm{~K}$. Полученные результаты находятся в согласии с данными работ [52,54,56]; отметим, что температура магнитного упорядочения для $\mathrm{HoBa}_{2} \mathrm{Cu}_{3} \mathrm{O}_{7-\delta}$ составляет $0.19 \mathrm{~K}$ [64].

Поскольку ниже будет анализироваться магнитосопротивление при температуре $4.2 \mathrm{~K}$, где парамагнитный (далее - РМ) вклад наибольший, заострим внимание на поведении петли гистерезиса намагниченности $M(H)$ при $T=4.2 \mathrm{~K}$ (рис. 4). По сравнению с „классической“ петлей гистерезиса сверхпроводника II рода, представленная на этом рисунке зависимость $M(H)$, далее $M_{\text {tot }}(H)$, имеет PM вклад. Наличие РМ вклада приводит к тому, что намагниченность становится положительной при увеличении внешнего поля уже в относительно небольших полях. Для того чтобы получить „невозмущенную“ сверхпроводящую (далее SP) петлю гистерезиса намагниченности, из экспериментальной зависимости $M_{\text {tot }}(H)$ вычиталась теоретическая зависимость намагниченности для парамагнетика $M_{\mathrm{PM}}(H)=N g J \mu_{\mathrm{B}} B_{J}\left(g J \mu_{\mathrm{B}} H / k T\right)$, где $N-$ количество магнитных ионов в единице объема, $\mu_{\mathrm{B}}-$ магнетон Бора, $k$ - константа Больцмана, $g$ - фактор Ланде, $J$ - квантовое число углового момента, $B_{J}-$ функция Бриллюэна. При разделении РМ и SP вкладов использовались значения для Но $(J=8, g=1.25)$, а концентрация магнитных ионов $N$ служила подгоночным параметром. Критерием адекватности разделения SP и РМ-вкладов служило отсутствие наклона SP петли гистерезиса в больших полях. Результат выделения РМ и SP вкладов показан на рис. 4. РМ-вклад соответствует концентрации гольмия в $\mathrm{HoBa}_{2} \mathrm{Cu}_{3} \mathrm{O}_{7-\delta}$, равной $2.32 \cdot 10^{21} \mathrm{~cm}^{-3}$ в малых полях и $3.01 \cdot 10^{21} \mathrm{~cm}^{-3}$ при $H=30 \mathrm{kOe}$. Эти величины меньше теоретического значения $5.79 \cdot 10^{21} \mathrm{~cm}^{-3}$, и подобное поведение наблюдалось при анализе систем $R e \mathrm{Ba}_{2} \mathrm{Cu}_{3} \mathrm{O}_{7-\delta}[52,54,56]$. Видимо, это вызвано частичной экранировкой от внешнего магнитного поля ионов Но внутри сверхпроводника. Скорее всего, РМ вклад возникает только от ионов Но, находящихся в нормальных сердцевинах вихрей Абрикосова и в приповерхностной области гранул с толщиной около глубины проникновения магнитного поля [54,56]. Построенная в результате разделения вкладов SP петля гистерезиса (рис. 4) имеет типичный для гранулярных ВТСП-вид.

Для определения плотности критического тока из петли гистерезиса использовалось соотношение, следующее из модели критического состояния [65], $J_{\mathrm{C}}\left(\mathrm{A} \cdot \mathrm{cm}^{-2}\right)=30 \Delta M\left(\mathrm{emu} \cdot \mathrm{cm}^{-3}\right) / d(\mathrm{~cm}), \quad$ где $\quad \Delta M=$ $=M\left(H_{\mathrm{dec}}\right)-M\left(H_{\mathrm{inc}}\right)-$ разница между убывающей и возрастающей ветвями гистерезиса, $d-$ характерный размер циркуляции тока. Для гранулярного ВТСП величина $d$ должна совпадать со средним размером гранул. Помимо данных сканирующей электронной микроскопии, величина $d$ может быть также оценена из асимметрии сверхпроводящей петли гистерезиса. Согласно результатам работы [66], $d \approx 2 \lambda /\left[1-\left(\Delta M /\left|2 M\left(H_{\text {inc }}\right)\right|\right)^{1 / 3}\right]$, где $\lambda-$ лондоновская глубина проникновения $(\lambda \approx 150 \mathrm{~nm}$ для системы $\mathrm{Y}-\mathrm{Ba}-\mathrm{Cu}-\mathrm{O}[67])$. Мы оценили $d \approx 3 \cdot 10^{-4} \mathrm{~cm} \mathrm{и}$ $J_{\mathrm{C}}=6.8 \cdot 10^{6} \mathrm{~A} \cdot \mathrm{cm}^{-2}$. Значение $d$ соответствует среднему размеру гранул в образце, см. п. 2, следовательно, полученная величина $J_{\mathrm{C}}-$ это максимальная плотность внутригранульного тока.

\section{2. Анализ гистерезиса $R(H)$. Вклады от мейсснеровских токов, вихрей Абрикосова и парамагнитной подсистемы в эффективное поле в межгранульной среде}

Зависимости $R(H)$ при $T=4.2 \mathrm{~K}$ для исследованного образца $\mathrm{HoBa}_{2} \mathrm{Cu}_{3} \mathrm{O}_{7-\delta}$ приведены на рис. 5. Приведены данные для двух величин транспортного тока: $I=180 \mathrm{~mA}$ (рис. 5, a) и $100 \mathrm{~mA}$ (рис. 5, $b$ ), полученные при циклировании внешнего поля до максимальных значений $H_{\max }= \pm 30 \mathrm{kOe}$ (рис. $5, a$ ) и $H_{\max }= \pm 10 \mathrm{kOe}$ (рис. 5,b). Стрелки на рисунке соответствуют направлению изменения внешнего поля; также для данных рис. 5, a показана начальная зависимость $R(H)_{\text {ini. }}$. 

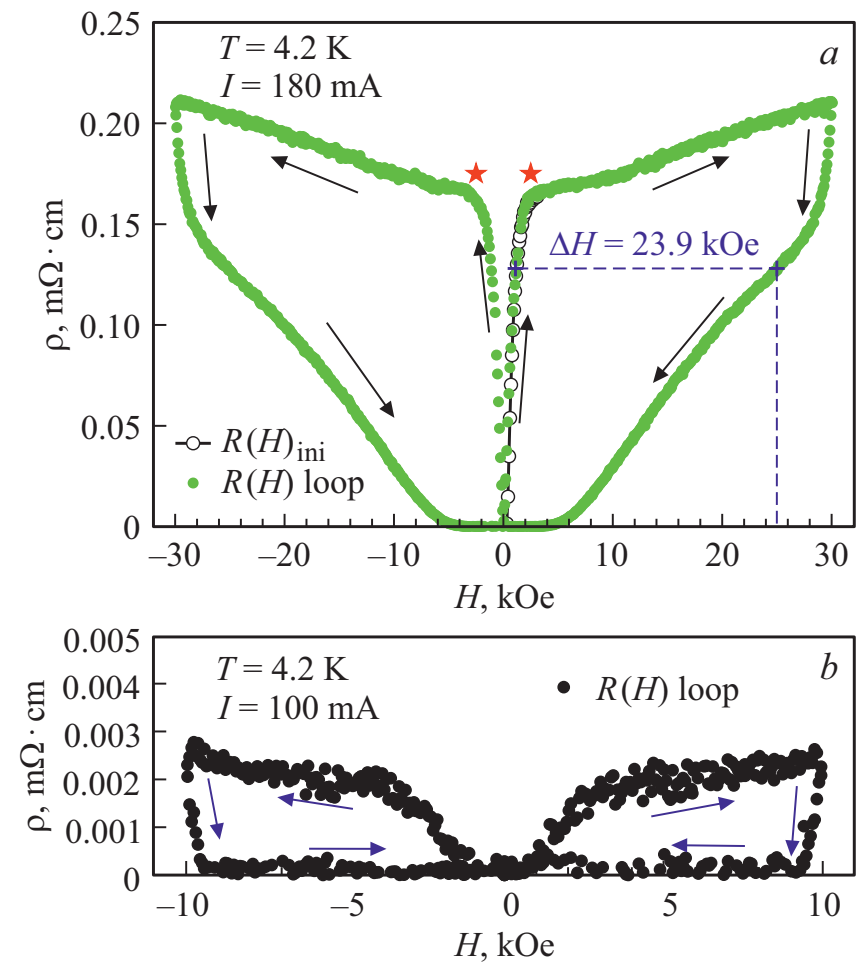

Рис. 5. Магнитосопротивление $R(H)$ образца $\mathrm{HoBa}_{2} \mathrm{Cu}_{3} \mathrm{O}_{7-\delta}$ при $T=4.2 \mathrm{~K}$ и указанных значениях транспортного тока $I$. Зависимости , $R(H)$ lоор“ соответствуют многократному циклированию поля до максимального значения $H_{\max }( \pm 30 \mathrm{kOe}(a)$ и $\pm 10 \mathrm{kOe}(b)), R(H)_{\text {ini }}$ - начальная зависимость магнитосопротивления. Стрелки на рисунке соответствуют направлению изменения внешнего поля. Горизонтальная пунктирная линия на $(a)$ иллюстрирует полевую ширину гистерезиса $R(H)$ при $H_{\text {dec }}=25 \mathrm{kOe}$.

Сразу отметим, что форма гистерезисной зависимости $R(H)$, относительное расположение ветвей магнитосопротивления $R\left(H_{\mathrm{inc}}\right)$ (поле возрастает) и $R\left(H_{\mathrm{dec}}\right)$ (поле убывает) подобны тому, что наблюдалось ранее для $\mathrm{YBa}_{2} \mathrm{Cu}_{3} \mathrm{O}_{7-\delta}[47-50]$.

Проанализируем полученные зависимости $R(H)$ в рамках концепции эффективного поля $B_{\text {eff }}$ в межгранульной среде, описанной во Введении. В различных теоретических представлениях магнитосопротивление сверхпроводника II рода является монотонной возрастающей функцией внешнего поля [68,4-12,51]. Для межгранульной среды магнитосопротивление функция $B_{\text {eff }}$, которое само зависит от внешнего поля. Следовательно, зависимость $B_{\text {eff }}(H)$ должна вести себя подобно зависимости $R(H)$. На рис. 6, a показана зависимость $B_{\text {eff }}(H)$, построенная по выражению (2) из экспериментальной зависимости $M_{\text {tot }}(H) \mathrm{HoBa}_{2} \mathrm{Cu}_{3} \mathrm{O}_{7-\delta}$ (рис. 4). Здесь, на основании результатов предыдущих работ на $\mathrm{YBa}_{2} \mathrm{Cu}_{3} \mathrm{O}_{7-\delta}$ [45-51], также был взят параметр $\alpha$, равный 25. Как видно из сравнения данных $R(H)$ (рис. 5, $a$ ) и $B_{\mathrm{eff}}(H)$ (рис. 6,a), согласия не наблюдается. Самое главное различие заключается в том, что гистерезис $R(H)$ идет „по часовой стрелке“ (для положительных значений $H)$, а гистерезис $B_{\text {eff }}(H)$ идет „Против часовой стрелки“. Отметим, что уменьшение значения параметра $\alpha$ не приводит к согласию в поведении зависимостей $R(H)$ и $B_{\text {eff }}(H)$ : гистерезис $B_{\text {eff }}(H)$ становится узким.

Петля магнитного гистерезиса сверхпроводника II рода возникает из-за циркуляции мейсснеровских токов и проникновения и пиннинга абрикосовских вихрей. Вклады от мейсснеровских токов и абрикосовских вихрей имеют различный знак относительно внешнего поля. Влияние абрикосовских вихрей и мейсснеровских токов на эффективное поле межгранульных границах также будет различным. На рис. 7, иллюстрирующем вышесказанное, схематично показаны линии магнитной индук-
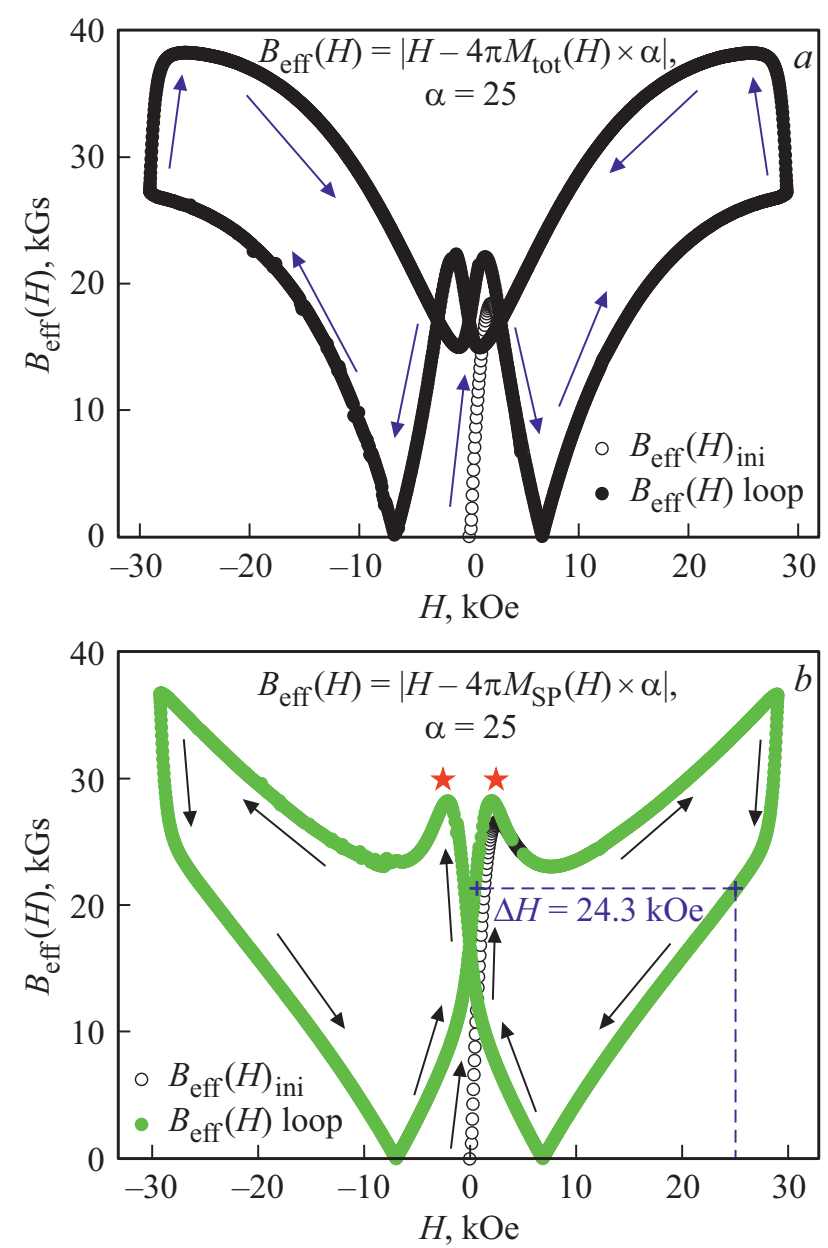

Рис. 6. Гистерезисная зависимость эффективного поля в межгранульной среде $B_{\text {eff }}(H)$, как функция внешнего поля $H$, построенная по выражению (2) из данных рис. 3 при $\alpha=25$ для $M(H)=M_{\text {tot }}(H)(a)$ и $M(H)=M_{\mathrm{SP}}(H)(b)$. Зависимость „B $B_{\text {eff }}(H)$ loор“ соответствует циклированию внешнего поля до $H_{\max }= \pm 30 \mathrm{kOe}$, зависимость $B_{\mathrm{eff}}(H)_{\mathrm{ini}}-$ начальный ход. Стрелки соответствуют направлению изменения внешнего поля. Обращает внимание различное направление гистерезиса для $(a)$ и $(b)$. Горизонтальная пунктирная линия иллюстрирует полевую ширину гистерезиса $B_{\text {eff }}(H)$ при $H_{\text {dec }}=25 \mathrm{kOe}$. 


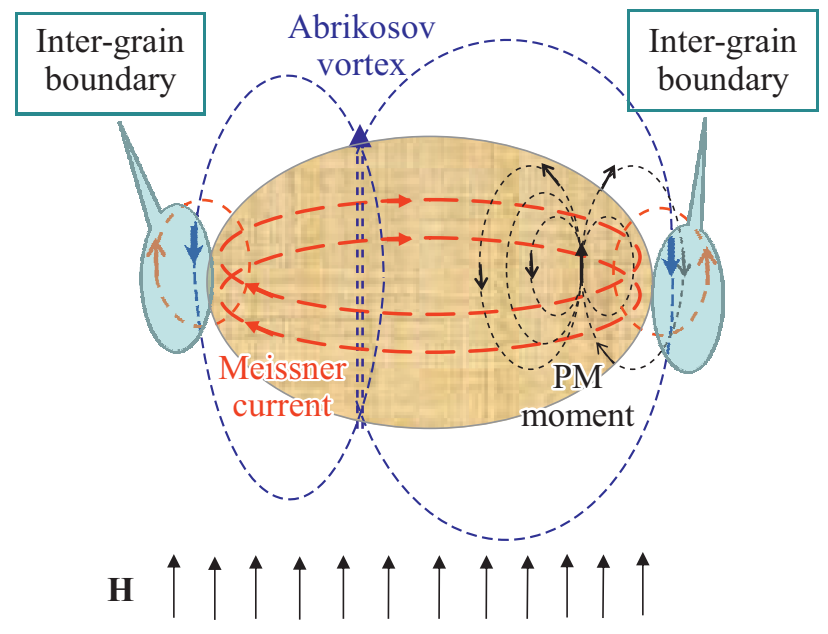

Рис. 7. Схематическое представление направлений линий магнитной индукции от вихрей Абрикосова, мейсснеровских токов и парамагнитных (РМ) моментов внутри и снаружи (межгранульные границы) гранулы в гранулярном ВТСП. Показан случай $H>0$.

ции от абрикосовских вихрей и мейсснеровских токов. Отметим, что „в чистом виде“ влияние абрикосовских

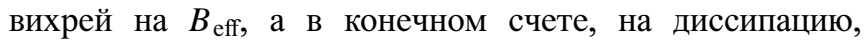
проявляется в наличии ненулевого сопротивления при $H=0$ после приложения внешнего поля [29,35,42-49], а также в релаксации остаточного сопротивления с течением времени (последнее связано с релаксацией намагниченности, вызванной выходом вихрей из сверхпроводника) [42,69]. В случае наличия парамагнитного вклада в ВТСП гранулах, также схематично показанного на рис. 7 (в виде одного спина), линии магнитной индукции от РМ моментов должны замкнуться, в том числе через межгранульную границу. Следовательно, вклад от РМ-моментов в эффективное поле $B_{\text {eff }}$ должен иметь знак такой же, как и от абрикосовских вихрей. Количественно вклад $M_{\mathrm{PM}}$ в общую намагниченность $M_{\text {tot }}(H)$ образца $\mathrm{HoBa}_{2} \mathrm{Cu}_{3} \mathrm{O}_{7-\delta}$ в больших полях достаточно большой, см. рис. 4 , он по величине превосходит вклад от сверхпроводящей подсистемы. Поэтому при подстановке $M_{\text {tot }}(H)$ в выражение (2) ожидаемые вклады от абрикосовских вихрей и РМ подсистемы превалируют над внешним полем и диамагнетизмом мейсснеровских токов. В результате зависимость $B_{\text {eff }}(H)$ имеет сильно немонотонный характер (рис. $6, a$ ), а гистерезис $B_{\text {eff }}(H)$ идет ,против часовой стрелки“, чего не наблюдается в эксперименте (рис. 5).

Будет логичным провести анализ гистерезиса $R(H)$ и построить зависимость $B_{\text {eff }}(H)$ без вклада РМ-подсистемы. Согласно рис. 4, $M_{\text {tot }}(H)=M_{\mathrm{SP}}(H)+M_{\mathrm{PM}}(H)$. Тогда, если в выражение (2) вместо $M(H)=M_{\text {tot }}(H)$ подставить только вклад от сверхпроводящей петли, $M(H)=M_{\mathrm{SP}}(H)$, полученная зависимость $B_{\text {eff }}(H)$ при $\alpha=25$ имеет вид, показанный на рис. $6, b$. В этом случае поведение зависимости $B_{\text {eff }}(H)$ уже хорошо опи- сывает гистерезис $R(H)$, и основанием этому является следующее. Ранее было показано, что гистерезисная зависимость $R(H)$ обладает универсальным и независимым от транспортного тока параметром полевой шириной гистерезиса, который определяется как величина отрезка $\Delta H=H_{\mathrm{dec}}-H_{\mathrm{inc}}$ при условии $R\left(H_{\mathrm{dec}}\right)=R\left(H_{\mathrm{inc}}\right)=$ const $[29,69,46,48,69-72]$. Длина горизонтальной штриховой линии на рис. 5, $a$ иллюстрирует величину этого параметра при $H_{\mathrm{dec}}=25 \mathrm{kOe}$. При этом $\Delta H=23.9 \mathrm{kOe}$, поскольку горизонтальная линия на рис. 5, $a$ пересекает ветку магнитосопротивления $R\left(H_{\text {inc }}\right)$ при значении $H_{\text {inc }}=1.1 \mathrm{kOe}$. Соответствующие величины намагниченности $M_{\mathrm{SP}}$ в полях $H_{\mathrm{dec}}=25 \mathrm{kOe}$ и $H_{\text {inc }}=1.1 \mathrm{kOe}$ показаны на рис. 4 (как „плюсы“, соединенные штриховой прямой). Горизонтальная штриховая линия на рис. $6, b$ имеет тот же смысл, что для рис. 5, $a$, но уже для зависимости $B_{\text {eff }}(H)$, и при том же значении $H_{\mathrm{dec}}=25 \mathrm{kOе}$ величина $\Delta H$ составляет $24.3 \mathrm{kOe}$, что очень близко к данным рис. 5, $a$. Такое согласие достигается только при достаточно большом значении параметра $\alpha=20-25$, что указывает на эффект сжатия магнитного потока в межгранульной среде (рис. 1). Сжатие потока проявляется в том, что для относительно небольших полей $H_{\text {inc }}$, несколько $\mathrm{kOe}$,

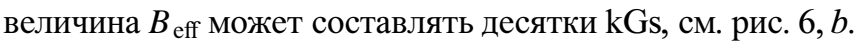
Отметим также, что на ветвях возрастающего поля зависимости $R\left(H_{\text {inc }}\right)$ проявляются аномалии в окрестности $H_{\text {inc }} \approx \pm 3 \mathrm{kOe}$, отмеченные символом звезды на рис. $5, a$. Эти аномалии соответствуют локальным максимумам зависимости $B_{\text {eff }}\left(H_{\text {inc }}\right)$ на рис. $6, b$ (звезды) и экстремумам зависимости $M_{\mathrm{SP}}(H)$ (рис. 3 ).

Итак, в качестве промежуточного вывода можно сказать, что анализ гистерезиса магнитосопротивления образца $\mathrm{HoBa}_{2} \mathrm{Cu}_{3} \mathrm{O}_{7-\delta}$ в рамках концепции эффективного поля в межгранульной среде показывает, что влияние РМ-подсистемы (магнитные моменты гольмия) практически не сказывается на величине эффективного поля в межгранульной среде, и, соответственно, на туннелировании носителей сверхпроводящего тока через межгранульные границы. В то же время, РМ-вклад в общую намагниченность образца достаточно большой, даже в умеренных полях (рис. 4). Для объяснения этого стоит вернуться к схематичному представлению линий магнитной индукции в межгранульной среде. Теперь, вместо одной (рис. 7), или двух (рис. 1) гранул рассмотрим сечение образца конечного размера (рис. 8), состоящего из множества гранул. Линии магнитной индукции от всех вкладов (мейсснеровских токов, абрикосовских вихрей, РМ моментов) должны замыкаться не только через границы между соседними гранулами, а также и через весь образец, поскольку из магнитных измерений все вклады хорошо видны, и только вихри, полностью замкнутые внутри образца, не будут давать вклад в полную намагниченность.

Вихри Абрикосова могут проходить через множество гранул. Поле вихря выходит за пределы сверхпроводящей гранулы в одной точке (диаметром около двух длин 


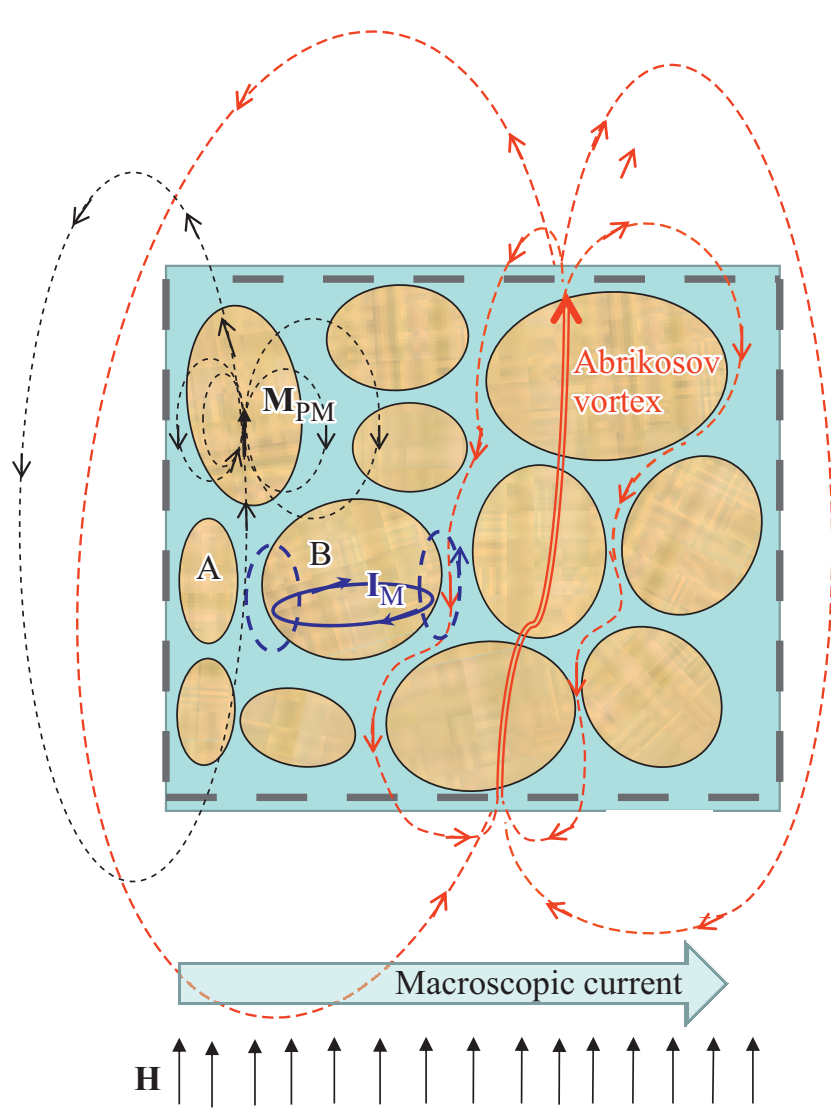

Рис. 8. Схематическое представление распределения линий магнитной индукции в гранулярном ВТСП „конечного размера“ (квадрат, ограниченный штриховыми прямыми). Овалы гранулы, пространство между ними - межгранульгная среда. Показаны вероятные траектории линий магнитной индукции от абрикосовских вихрей, мейсснеровского тока $\mathbf{I}_{\mathrm{M}}$, и парамагнитного момента $\mathbf{M}_{\mathrm{PM}}$. Направления линий магнитной индукции соответствуют случаю $H>0$.

когерентности). Линии магнитной индукции от вихря Абрикосова, вышедшие из верхней гранулы, должны замыкаться в одном месте - в „начале“ этого же вихря на самой нижней грануле (см. рис. 8). Линии магнитной индукции от вихря или связки вихрей Абрикосова могут замыкаться как снаружи образца, так и через межгранульные границы. Из общих представлений электродинамики ясно, что наибольшая плотность линий магнитной индукции будет вокруг гранулы, из которой выходит вихрь, т.е. в ближайших межгранульных промежутках. Это проявляется как сжатие потока в межгранульной среде, применительно к подсистеме абрикосовских вихрей.

Мейсснеровские токи $\left(\mathbf{I}_{\mathrm{M}}\right)$ текут в плоскостях, перпендикулярных внешнему полю, и магнитное поле от них эквивалентно полю замкнутого кругового тока. Линии этого поля должны проходить через весь образец, что проявляется как диамагнетизм сверхпроводящего образца. Однако наибольшая величина поля, индуцированного мейсснеровскими токами, будет вблизи края гранулы, т.е. вблизи межгранульной границы, и тогда в геометрии $\mathbf{H} \perp \mathbf{I}_{\text {macro }}\left(\mathbf{H} \perp \mathbf{I}_{\mathrm{m}}\right)$ для туннелирования, например, из гранулы А в гранулу В (см. рис. 8) поле от мейсснеровского тока будет параллельно внешнему полю. Тогда в границе между гранулами А и В можно говорить о сильном влиянии поля от мейсснеровского тока, что укладывается в рамки „сжатия магнитного потока“ в межгранульной среде и обуславливает достаточно большую величину параметра $\alpha$ для описания гистерезиса $R(H)$.

Магнитные моменты редкоземельного элемента (гольмия) ведут себя как независимая РМ-подсистема, о чем свидетельствуют результаты работ $[52,54,56]$ и результаты данной работы (рис. 3,4 , см. п. 3.1). Линии магнитной индукции от РМ моментов также могут замыкаться и через весь образец, и в локальных областях, как выходя из гранулы, так и не выходя из нее. Такая картина схематично показана на рис. 8. Если эти линии магнитной индукции не концентрируются в межгранульных промежутках, подобно линиям магнитной индукции от вихрей Абрикосова, то РМ-вклад должен влиять на поле в межгранульных границах в гораздо меньшей степени. Следовательно, полученный выше „отрицательный результат“", в котором нельзя объяснить гистерезис $R(H)$ в рамках выражения (2) с использованием намагниченности $M_{\text {tot }}(H)$ свидетельствует о том, что линии магнитной индукции от РМ моментов могут замыкаться, проходя через множество гранул. Тогда концентрации в межгранульной среде не происходит.

С другой стороны, спины, расположенные близко к краю гранулы, все равно должны давать вклад в эффективное поле в межгранульной границе, хотя и без эффекта сжатия магнитного потока. Чтобы учесть эту возможность, перепишем выражение (2) в следующем виде:

$$
B_{\text {eff }}(H)=\left|H-4 \pi M_{\mathrm{SP}}(H) \times \alpha_{\mathrm{SP}}-4 \pi M_{\mathrm{PM}}(H) \alpha_{\mathrm{PM}}\right| .
$$

В выражении (3) учтено, что $M_{\mathrm{tot}}(H)=M_{\mathrm{SP}}(H)$ $+M_{\mathrm{PM}}(H), \alpha_{\mathrm{SP}}=\alpha$, а $\alpha_{\mathrm{PM}}-$ параметр, характеризующий возможное сжатие линий магнитной индукции от РМ-моментов. Рис. 9 содержит зависимости $B_{\text {eff }}(H)$, вычисленные по выражению (3) из данных $M_{\mathrm{SP}}(H)$ и $M_{\mathrm{PM}}(H)$, приведенных на рис. 4 , при величинах $\alpha_{\mathrm{SP}}=25$ (как и ранее) и $\alpha_{\mathrm{PM}}$, равных $0,1,3,5$. При $\alpha_{\mathrm{PM}}=0$ зависимость $B_{\text {eff }}(H)$ воспроизводит данные рис. $5, b$, и как было показано выше, есть хорошее согласие с величиной ширины гистерезиса $\Delta H$ для данных $R(H)$ (см. рис. $6, b$ и $5, a)$. В принципе, для зависимости $B_{\mathrm{eff}}(H)$ при $\alpha_{\mathrm{PM}}=1$ значение параметра $\Delta H$ при $H_{\mathrm{dec}}=25 \mathrm{kOe}$ составляет $24.8 \mathrm{kOe}$ (горизонтальная линия $B_{\text {eff }}(H)=$ const пересекается с зависимостью $B_{\text {eff }}\left(H_{\text {inc }}\right)$ при $\left.H_{\text {inc }}=0.2 \mathrm{kOe}\right)$, что также близко величине $\Delta H$ зависимости $R(H)$ $(23.9 \mathrm{kOe}$, см. рис. $5, a)$. При дальнейшем увеличении $\alpha_{\mathrm{PM}}$, как видно из рис. 9, горизонтальная штриховая линия $B_{\text {eff }}\left(H_{\mathrm{dec}}=25 \mathrm{kOe}\right)=$ const уже не пересекает ветку $B_{\text {eff }}\left(H_{\text {inc }}\right)$, а пересекает спадающего поля $B_{\text {eff }}\left(H_{\text {dec }}\right)$, 


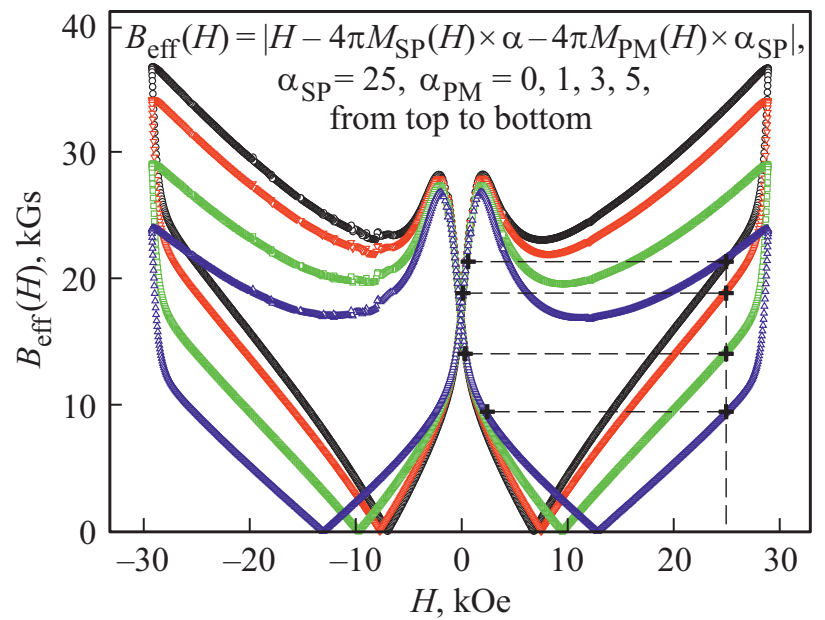

Рис. 9. Зависимости эффективного поля в межгранульной среде $B_{\text {eff }}(H)$, как функции внешнего поля $H$, построенные по выражению (3) из данных рис. 3 для разных значений $\alpha_{\mathrm{PM}}$. Горизонтальные пунктирные линии иллюстрируют полевую ширину гистерезиса $B_{\mathrm{eff}}(H)$ при $H_{\mathrm{dec}}=25 \mathrm{kOe}$.

что плохо согласуется с поведением зависимости $R(H)$ (рис. 5, $a$ ). Другим проявлением несогласия с поведением гистерезиса магнитосопротивления является достаточно сильный сдвиг минимума зависимости $B_{\text {eff }}\left(H_{\mathrm{dec}}\right)$

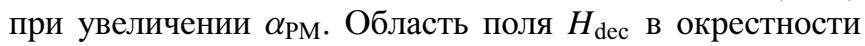
этого минимума соответствует нулевому сопротивлению зависимости $R\left(H_{\mathrm{dec}}\right)$, см. рис. 5 , и при $\alpha_{\mathrm{PM}}=3$ и 5 значение поля минимума зависимости $B_{\mathrm{eff}}\left(H_{\mathrm{dec}}\right)$ значительно превосходит область „, $R\left(H_{\mathrm{dec}}\right)=0$, перименте. Следовательно, если РМ подсистема влияет на поле в межгранульной среде, то это влияние незначительно. Можно говорить, что согласие в поведении экспериментальной зависимости $R(H)$ и зависимости $B_{\text {eff }}(H)$ наблюдается только при значении параметра $\alpha_{\mathrm{PM}}$ около единицы. Это указывает на то, что сжатия линий магнитной индукции от РМ-моментов в межгранульной среде не происходит.

\section{4. Выводы}

В гранулярном ВТСП НоBa $\mathrm{Cu}_{3} \mathrm{O}_{7-\delta}$ магнитные моменты атомов гольмия ведут себя как независимая парамагнитная подсистема. Анализ гистерезиса магнитосопротивления в рамках концепции эффективного поля в межгранульной среде (выражение (2)) показал, что парамагнитная подсистема практически не оказывает влияние на эффективное поле в межгранульной среде. Это указывает на принципиально различный характер перераспределения линий магнитной индукции в гранулярном ВТСП от „сверхпроводящего отклика“ (мейсснеровские токи и вихри Абрикосова) и парамагнитных моментов атомов гольмия. В межгранульной среде происходит концентрация линий магнитной индукции от мейсснеровских токов и вихрей Абрикосова. Такое сжа- тие потока приводит к тому, что поле в межгранульной среде значительно отличается от внешнего поля, и в определенных условиях (при возрастании внешнего поля) может на порядок превышать его. Линии магнитной индукции от парамагнитных моментов не концентрируются в межгранульной среде, а замыкаются, проходя через ВТСП гранулы. Влияние парамагнитных моментов на эффективное поле в межгранульной среде незначительно. Количественный анализ парамагнитного вклада в общую намагниченность ВТСП образца $\mathrm{HoBa}_{2} \mathrm{Cu}_{3} \mathrm{O}_{7-\delta}$ показывает, что этот вклад обусловлен магнитными моментами гольмия, находящимися в сердцевинах вихрей Абрикосова и в приповерхностных областях гранул с толщиной порядка глубины проникновения магнитного поля. Следовательно, линии магнитной индукции от магнитных ионов замыкаются, проходя через эти области.

Мы посвящаем эту работу памяти Виталия Александровича Финкеля (Харьковский физико-технический институт), ушедшего в марте 2021 г. Цитированные здесь и другие его работы важны для понимания механизмов магнитосопротивления гранулярных сверхпроводников.

\section{Благодарности}

Авторы благодарят В.М. Соснина, К.А. Шайхутдинова, А.А. Красикова за обсуждение результатов, И.В. Немцева за электронную микроскопию образца.

\section{Финансирование работы}

Исследование выполнено при финансовой поддержке Российского фонда фундаментальных исследований, Правительства Красноярского края, Красноярского краевого фонда науки в рамках научного проекта № 20-42-240008 „Влияние внедрения парамагнитных ионов редкоземельных элементов на сверхпроводящие свойства материалов на основе ҮВСО“.

Исследование микроструктуры и часть магнитных измерений были проведены с использованием оборудования Центра коллективного пользования ФИЦ КНЦ СО РАН.

\section{Конфликт интересов}

Авторы заявляют, что у них нет конфликта интересов.

\section{Список литературы}

[1] M.A. Dubson, S.T. Herbet, J.J. Calabrese, D.C. Harris, B.R. Patton, J.C. Garland. Phys. Rev. Lett. 60, 1061 (1988).

[2] L. Ji, M.S. Rzchowski, N. Anand, M. Tinkham. Phys. Rev. B 47, 470 (1993).

[3] M. Prester. Supercond. Sci. Technol. 11, 333 (1998).

[4] A.C. Wright, K. Zhang, A. Erbil. Phys. Rev. B. 44, 863 (1991).

[5] C. Gaffney, H. Petersen, R. Bednar. Phys. Rev. B. 48, 3388 (1993).

[6] H.S. Gamchi, G.J. Russell, K.N.R. Taylor. Phys. Rev. B. 50, 12950 (1994). 
[7] R.J. Soulen, T.L. Francavilla, W.W. Fuller-Mora, M.M. Miller, C.H. Joshi, W.L. Carter, A.J. Rodenbush, M.D. Manlief, D. Aized. Phys. Rev. B 50, 478 (1994).

[8] D.H. Liebenberg, R.J. Soulen, T.L. Francavilla, W.W. Fuller-Mora, P.C. Mc Intyre, M.J. Cima. Phys. Rev. B 51, 11838 (1995).

[9] R.J. Soulen, T.L. Francavilla, A.R. Drews, L. Toth, M.S. Osofsly, W.L. Lechter, E.F. Skelton. Phys. Rev. B 51, 1393 (1995).

[10] W.M. Tieran, R. Joshi, R.B. Hallock. Phys. Rev. B 48, 3423 (1993).

[11] Y. Zhao, X.B. Zuge, J.M. Xu, L. Cao. Phys. Rev. B 49, 6985 (1994).

[12] L. Urba, C. Acha, V. Bekeris. Physica C 279, 95 (1997).

[13] E. Hannachi, M.K. Ben Salem, Y. Slimani, A. Hamrita, M. Zouaoui, F. Ben Azzouz, M. Ben Salem. Physica B 430, 52 (2013).

[14] E. Hannachi, Y. Slimani, A. Ekicibil, A. Manikandan, F. Ben Azzouz. J. Mater. Sci.: Mater. Electron. 30, 8805 (2019).

[15] Y. Slimani, E. Hannachi, A. Hamrita, M.K. Ben Salem, M. Zouaoui, M. Ben Salem, F. Ben Azzouz. J. Supercond. Nov. Magn. 28, 487 (2015).

[16] S. Shifang, Z. Yong, P. Guoqian, Y. Daoq, Z. An, C. Zuyao, Q. Yitai, K. Eiyan, Z. Qirui. Europhys. Lett. 6, 4, 359 (1988).

[17] Y.J. Quian, Z.M. Tang, K.Y. Chen, B. Zhou, J.W. Qui, B.C. Miao, Y.M. Cai. Phys. Rev. B 39, 4701 (1989).

[18] P. Múne, F.C. Fonseca, R. Muccillo, R.F. Jardim. Physica C 390, 363 (2003).

[19] C.A.M. dos Santos, M.S. da Luz, B. Ferreira, A.J.S. Machado. Physica C 391, 345 (2003).

[20] Н.Д. Кузьмичев. Письма в ЖЭТФ 74, 291 (2001).

[21] Н.Д. Кузьмичев. ФТТ 43, 1934 (2001).

[22] Т.В. Сухарева, В.А. Финкель. ЖЭТФ 134, 922 (2008).

[23] Т.В. Сухарева, В.А. Финкель. ФТТ 50, 6, 961 (2008).

[24] В.В. Деревянко, Т.В. Сухарева, В.А. Финкель. ЖТФ 78, 36 (2008).

[25] Т.В. Сухарева, В.А. Финкель. ФТТ 52, 3, 424 (2010).

[26] M.E. McHenry, P.P. Maley, J.O. Willis. Phys. Rev. B 40, 2666 (1989).

[27] E. Altshuler, J. Musa, J. Barroso, A.R.R. Papa, V. Venegas. Cryogenics 33, 308 (1993).

[28] J. López, P. Múne, S. Garsía, E. Altshuler. Physica C 272, 13 (1996).

[29] Д.А. Балаев, Д.М. Гохфельд, А.А. Дубровский, С.И. Попков, К.А. Шайхутдинов, М.И. Петров. ЖЭТФ 132, 1340 (2007).

[30] A.V. Mitin. Physica C 235-240, 3311 (1994).

[31] D. López, F. de la Cruz . Phys. Rev. B 43, 13, 11478 (1991).

[32] D. López, R. Decca, F. de la Cruz. Supercond. Sci. Technol. 5, S276 (1992).

[33] J.L. Giordano, J. Luzuriaga, A. Badía-Majós, G. Nieva, I. Ruíz-Tagle. Supercond. Sci. Technol. 19, 385 (2006).

[34] Д.А. Балаев, А.А. Быков, С.В. Семенов, С.И. Попков, А.А. Дубровский, К.А. Шайхутдинов, М.И. Петров. ФТТ 53, 5, 865 (2011).

[35] С.В. Семенов, Д.А. Балаев, М.А. Почекутов, Д.А. Великанов. ФТТ 59, 7, 1267 (2017).

[36] Э.Б. Сонин. Письма ЖЭТФ 47, 415 (1988).

[37] M. Mahel'. J. Pivarč, Physica C 308, 147 (1998).

[38] O.V. Gerashchenko, S.L. Ginzburg. Supercond. Sci.Technol. 13, 332 (2000).

[39] M.S. da Luz, C.A.M. dos Santos, B. Ferreira, A.J.S. Machado. Physica C 408-410, 460 (2004).
[40] А. Суханов, В. Омельченко. ФНТ 30, 604 (2004).

[41] D. Daghero, P. Mazzetti, A. Stepanescu, P. Tura. Phys. Rev. B 66, 13, 11478 (2002).

[42] D.A. Balaev, S.I. Popkov, E.I. Sabitova, S.V. Semenov, K.A. Shaykhutdinov, A.V. Shabanov, M.I. Petrov. J. Appl. Phys. 110, 093918 (2011).

[43] D.A. Balaev, S.V. Semenov, M.I. Petrov. J. Supercond. Nov. Magn. 27, 1425 (2014).

[44] Д.А. Балаев, С.И. Попков, К.А. Шайхутдинов, М.И. Петров, Д.М. Гохфельд. ФТТ 56, 8, 1492 (2014).

[45] D.A. Balaev, S.V. Semenov, M.A. Pochekutov. J. Appl. Phys. 122, 123902 (2017).

[46] S.V. Semenov, D.A. Balaev. Physica C 550, 19 (2018).

[47] S.V. Semenov, D.A. Balaev. J. Supercond. Nov. Magn. 32, 2409 (2019).

[48] С.В. Семенов, Д.А. Балаев. ФТТ 62, 7, 1008 (2020).

[49] D.A. Balaev, S.V. Semenov, D.M. Gokhfeld. J. Supercond. Nov. Magn. 34, 1067 (2021).

[50] С.В. Семенов, Д.А. Балаев, М.И. Петров. ФТТ 63, 7, 854 (2021).

[51] S.V. Semenov, A.D. Balaev, D.A. Balaev. J. Appl. Phys. 125, 033903 (2019).

[52] H. Theuss, H. Kronmüller. Physica C 242, 155 (1995).

[53] M.R. Koblischka, M. Murakami. J. Supercond.: Incorporat. Nov. Magn. 14, 3, 415 (2001).

[54] E. Altin, D.M. Gokhfeld, F. Kurt, Z.D. Yakinci. J. Mater. Sci: Mater. Electron. 24, 5075 (2013).

[55] A. Öztürk, M. Dogăn, Í. Düzgün, S. Celebi. J. Supercond. Nov. Magn. 29, 1787 (2016).

[56] D.M. Gokhfeld, D.A. Balaev, I.S. Yakimov, M.I. Petrov, S.V. Semenov. Ceram. Int. 43, 9985 (2017).

[57] B.W. Lee, J.M. Ferreira, Y. Dalichaouch, M.S. Torikachvili, K.N. Yang, M.B. Maple. Phys. Rev. B 37, 4, 2368 (1988).

[58] А.Д. Балаев, Ю.В. Бояршинов, М.М. Карпенко, Б.П. Хрусталев. ПТЭ 3, 167 (1985).

[59] D.A. Balaev, A.G. Prus, K.A. Shaykhutdinov, D.M. Gokhfeld, M.I. Petrov. Supercond. Sci. Technol. 20, 495 (2007).

[60] В.В. Деревянко, Т.В. Сухарева, В.А. Финкель. ФТТ 60, 3, 465 (2018)

[61] Т.В. Сухарева, В.А. Финкель. Письма ЖЭТФ 108, 4, 249 (2018).

[62] Т.В. Сухарева, В.А. Финкель. ФНТ 44, 3, 258 (2018).

[63] Т.В. Сухарева, В.А. Финкель. ФНТ 46, 5, 653 (2020).

[64] K. Osuch. Physica C 383, 263 (2002).

[65] C.P. Bean. Rev. Mod. Phys. 36, 31 (1964).

[66] Д.М. Гохфельд. Письма ЖТФ 45, 2,3 (2019).

[67] D.N. Zheng, A.M. Campbell, J.D. Johnson, J.R. Cooper, F.J. Blunt, A. Porch, P.A. Freeman. Phys. Rev. B 49, 2, 1417 (1994).

[68] G. Blatter, M.V. Feigel'man, V.B. Gekshkebein, A.I. Larkin, V.M. Vinokur. Rev. Mod. Phys. 66, 4, 1125 (1994).

[69] Д.А. Балаев, А.А. Дубровский, К.А. Шайхутдинов, С.И. Попков, Д.М. Гохфельд, Ю.С. Гохфельд, М.И. Петров. ЖЭТФ 135, 271 (2009).

[70] Д.А. Балаев, А.А. Дубровский, С.И. Попков, Д.М. Гохфельд, С.В. Семенов, К.А. Шайхутдинов, М.И. Петров. ФТТ 54, 11, 11 (2012).

[71] Д.А. Балаев, С.В. Семенов, М.И. Петров. ФТТ 55, 12, 2305 (2013).

[72] A. Altinkok, K. Kilic, M. Olutas, A. Kilic. J. Supercond. Nov. Magn. 26, 3085 (2013).

Редактор Т.Н. Василевская 